\title{
The expansion of the Universe may be an illusion created by Compton scattering of free electrons
}

\author{
GuanFeng Cheng* \\ Hefei Jinlida thermal equipment China
}

\begin{abstract}
The two most precise gauges of the Universe's expansion rate have recently been in glaring disagreement. The measurement of the Hubble parameter makes the Universe expansion theory more and more confusing, the cosmological redshift may not only be related to distance but also to other factors, and the expansion of the Universe may be just an illusion. The Compton effect of free electrons and low energy photons has been observed in the laboratory. This paper presents a theory: Free electron Compton scattering (FEC) may produce the illusion of the Universe expansion: Photons interact with a large number of free electrons on their way to us from a distant source. FEC causes photons to redshift (FEC redshift), and the photon beam expands along the propagation direction, these may produce the illusion of cosmic expansion (FEC cosmic model). FEC redshift is independent of wavelength, and proportional to distance and corresponding electron density, consistent with the Doppler effect of the expansion of the Universe. Flare redshift is a common phenomenon, and it is difficult to use the Doppler effect to explain alone, there is evidence of FEC redshift in flare redshift.
\end{abstract}

Keywords: Universe expansion, Hubble parameter, free electron Compton scattering, flare redshift.

\section{Introduction}

In 1927, the Belgian astronomer and cosmologist Georges Lemaître first proposed the Big Bang hypothesis. In 1929, the American astronomer Hubble proposed Hubble's law based on the redshift of galaxies being proportional to the distance, and deduced the theory of the expansion of the universe that galaxies are far away from each other. In 1946, American physicist Gamow formally proposed the big bang theory. The explosion theory believes that the universe was formed by a big explosion that occurred about 14 billion years ago. At the end of the last century, observations of the Ia supernova showed that the universe was accelerating, and the energy for accelerating expansion came from dark energy. The Big Bang theory successfully predicted the microwave background radiation (CMBR) and cosmic abundance, which became an important support for the theory of cosmic expansion. The theory of Universe expansion has been widely recognized by the scientific community.

There are also many problems with the theory of expanding universe, such as the problem that the Hubble constant is not a constant, the problem that dark energy is nowhere to be found. For much of this decade, the two most precise gauges of the Universe's rate of expansion have been in glaring disagreement. The measurement of the Hubble parameter makes the theory of cosmic expansion more and more confusing ${ }^{1-4}$. 
The results from different samples are also different ${ }^{3,5,6}$. Riess et al. ${ }^{6}$ Present an improved determination of the Hubble constant from Hubble Space Telescope (HST) observations of 70 long-period Cepheids in the Large Magellanic Cloud and give the latest value of $74 \pm 1.42 \mathrm{~km} \mathrm{~s}^{-1} \mathrm{Mpc}^{-1}$ with an error margin of just $1.91 \%$. Freedman et al. ${ }^{3}$ find a value of $6.9 \pm 0.8( \pm 1.1 \%$ stat $) \pm 1.7( \pm 2.4 \%$ sys $) \mathrm{km} \mathrm{s}^{-1} \mathrm{Mpc}^{-1}$ based on a calibration of the tip of the red giant branch (TRGB) applied to Type Ia supernovae (SNE Ia) with a sample containing about 100 well-observed SNe Ia. The Hubble parameter based on Quasar is larger ${ }^{5}$. There is the systematic discrepancy in the Hubble parameter of different types of stars and means that the Hubble parameter is not only related to the distance but also to the type of star considered. The expansion rate of the Universe indirectly arising from the CMBR is $67.8 \mathrm{~km} \mathrm{~s}^{-1} \mathrm{Mpc}^{-1}$. These most precise Hubble measurements to date bolster the idea that new physics may be needed to explain the mismatch ${ }^{3,5-7}$.

The point of view of this paper: Based on the fact that the big bang is incredible; the dark energy is nowhere to be found; and if the Hubble parameter is not only related to distance, but also to the type of star, then Hubble's law is only an approximation, and the Universe expansion theory based on Hubble's law may be wrong, and the expansion of the Universe may be just an illusion. The "new physics" may be: the illusion of the Universe expansion created by Compton scattering of free electrons.

Whether the Universe really expands has been controversial. The first alternate suggestion ("Tired light") was made by Zwicky (1929) where he proposed that: redshifts are explained as arising not due to the expansion of the Universe, but by the action of an intervening medium that causes photon energies to decrease as the photons from distant objects propagate through intergalactic space toward the Earth. "Tired light" is unphysical because of the following ${ }^{8,9}: 1$. Any scattering process with energy transfer from the photon beam to the scattering medium, as required for a redshift, must broaden (deflect) the beam. This effect would cause images of distant galaxies to be fuzzier than their local counterparts, which they are not. 2. Time dilation of supernova light curves ${ }^{10,11} .3$. The relationship between the spectrum of the cosmic microwave background and its energy density. There is no photon dilution mechanism in "tired light", and the photon density of CMBR derived is $(1+z)^{3}$ times larger than the observed value. 4. The Tolman Surface Brightness Test for the Reality of the Expansion: "the surface brightness of a set of standard" (identical) objects will decrease by $(1+z)^{4}$. One factor of $(1+z)$ comes from the decrease in the energy of each photon due to the redshift. The second factor comes from the decrease in the number-flux per unit time. Two additional factors of $(1+z)$ come from the apparent increase of area due to aberration ${ }^{12}$. For more details, see: Edward L. Wright's "Errors in Tired Light"

Compton models are in the class of "tiled light" cosmic models. Compton scattering usually refers to the interaction of high-energy photons and bound electrons, which may produce a large scattering angle. This leads no one to think that the redshift of cosmology is caused by Compton scattering. 
FEC refers to Compton scattering between free electrons and low-energy (low-frequency) photons. The characteristic spectral line mainly exists in low-frequency band. The electron is free, not bound. If FEC scattering exists, the scattering angle is very small, and the propagation direction of the photon is almost unchanged.

When the interaction is between relatively cold CMB photons and hot free electrons, it is known as the thermal Sunyaev-Zel'dovich effect (Inverse Compton effect), the cold CMB photons are blueshifted ${ }^{13,14}$. Will relatively hot photons be redshifted by relatively cold free electrons?

Nonlinear Compton scattering is encountered when several photons from a strong laser beam are scattered by a free electron to produce a photon of different energy, this process has been calculated theoretically and successfully measured ${ }^{15-19}$. These show that low-energy photons have not only wave properties but also particle properties, and mean that Compton scattering may occur regardless of the interaction of high-energy or low-energy photons with free electrons. Therefore FEC effect exists, the Compton scattering angle of FEC exists. This scattering angle produces the magic effect: photon redshift and photon beam expansion, which makes FEC cosmic model different from "tiled light" cosmic models.

\section{FEC cosmic model}

FEC causes photons to redshift (See below for details), and the photon beam expands along the propagation direction, producing the illusion of cosmic expansion (FEC cosmic model. See FIG. 1). The Compton effect of low-energy photons and free electrons is very weak and neglected. Free electrons make the photons move forward in slight jitter, photons jitter causes the photon beam to expand. The expansion of the photon beam is proportional to redshift $z$, showing the same astronomical phenomena as the expansion of the Universe.

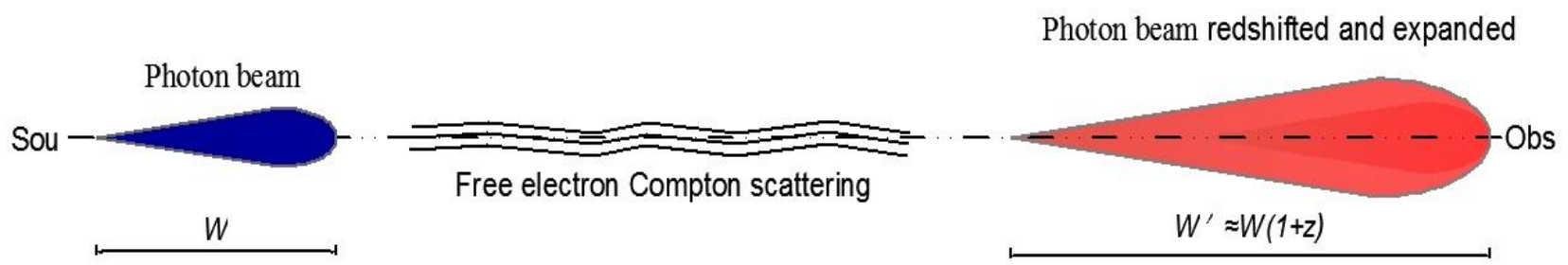

FIG. 1: FEC cosmic model: Free electron Compton scattering (FEC). FEC causes photons to redshift and the photon beam expands. Where $W$ is the width (time) of the photon beam. FEC create the illusion that the universe expands.

FEC scattering angle $\theta$ is very small, when a photon interacts with a large number of electrons, the random scattering angles cancel each other. The photons move along the propagation direction in the jitter which is not easy to observe. The scattering angle to the observer is $\sum \theta$, the probability distribution of $\sum \theta$ is a 
Gaussian distribution, the axis $\left(\sum \theta=0\right)$ is the line connecting the light source and the telescope. Therefore, FEC does not blur the image of distant galaxies. Since the scattering angle $\theta$ occurs randomly in a particular range, the size and direction are different, leading to the inconsistent trajectories of photons and the photon beam expanding. The photon energy decreases $(1+z)$ times, the width $\mathrm{W}$ (time) of the photon beam is stretched $(1+z)$ times, and the photon density decreases $(1+z)^{3}$. The effect is as described in "the Tolman Surface Brightness Test".

CMBR is almost perfect $2.725 \mathrm{~K}$ blackbody radiation ${ }^{20}$. The big bang theory successfully predicts CMBR and the relationship ${ }^{21,22}$ of $T_{C M B}(Z)=T_{C M B}^{0}(1+Z)$.

If CMBR was not formed by the Big Bang, how was CMBR formed? The assumption of this paper is: CMBR $\left(T_{C M B}^{0}\right)$ is mainly created by large-scale interstellar medium (Mainly Interstellar neutral atoms, molecules, dust) radiation $\left(T_{C M B}(Z)\right)$, due to the FEC effect, the photon density of CMBR $\left(T_{C M B}(Z)\right)$ is diluted by $(1+z)^{3}$ times and the temperature is reduced by $(1+z)$ times. The temperature of the interstellar medium is about $10 \sim 100 \mathrm{~K}$. The interstellar medium in the Universe is almost uniform and isothermal, and local is transparent, when the distance is greater than $z=4.7$, the interstellar medium gradually formed a fog and progressively made the Universe opaque, forming a blackbody with a temperature of $T_{C M B}(Z)$. In this way, CMBR comes from the distant Universe $^{8}(z>4.7)$.

Most of the energy of starlight is absorbed by the intergalactic and the interstellar medium, and a small part of it becomes microwave background radiation. The intergalactic medium and the interstellar medium absorb the radiation energy and emission radiation. CMBR is formed indirectly by starlight, which is diluted and redshifted by the FEC effect.

It can be derived from the FEC cosmic model that the emissivity of CMBR is greater than 1 . The emissivity of the Universe expansion theory will be less than 1 due to space expansion. The best fit emissivity is $1.09^{23}$. The number of faint radio sources of the FEC model will increase, and of cosmic expansion mode will decrease and dilute. The number of faint radiation sources can distinguish FEC model and the Universe mode. For more details, see: Edward L. Wright's "Errors in the Steady State and Quasi-SS Models".

\section{FEC redshift}

FIG.2.The interaction between free electrons and photons: the electrons are affected by the force $\vec{F}$ of electromagnetic radiation, $\vec{F}=\overrightarrow{F_{E}}+\overrightarrow{F_{B}}, \overrightarrow{F_{B}}=\vec{V} \times \vec{B}, \vec{V}$ can be regarded as the current and displacement current generated by the movement of electrons. When the initial velocity of electron is neglected, the electron is always affected by the electric field force $\overrightarrow{F_{E}}$ and moves perpendicular to the $\vec{k}$-direction, so the electron always suffers recoil in the $\vec{k}$-direction by $\overrightarrow{F_{B}}$, and Photon recoil is opposite to the $\vec{k}$-direction. When the photon is low-energy, $\overrightarrow{F_{B}} \square \overrightarrow{F_{E}}, \overrightarrow{F_{B}}$ can be ignored, the electron recoil and the photon recoil are ignored because they are too small, the process is called Thomson scattering .Otherwise, it is called Compton scattering. 


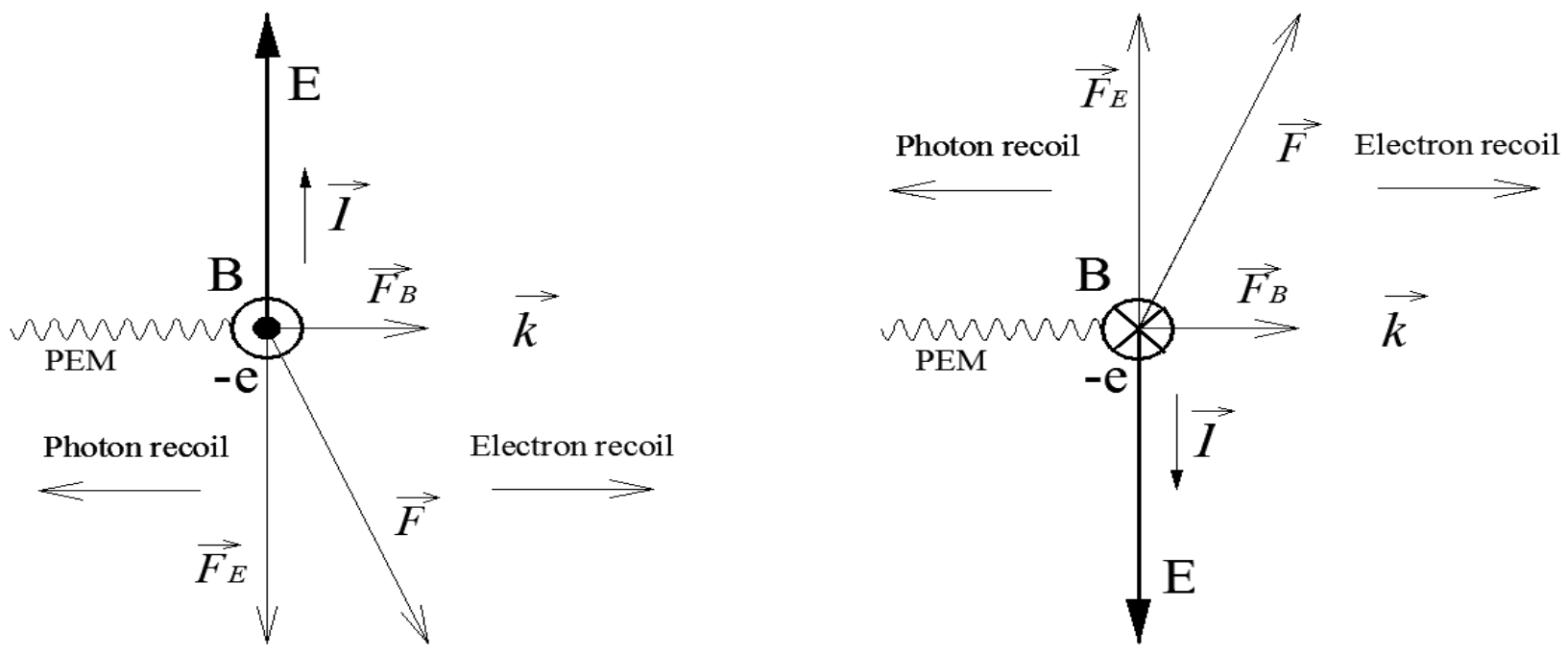

FIG.2: Photon PEM (plane electromagnetic wave) interacts with a free electron. Where $\mathrm{E}$ is the photon's electric field, B is the photon's magnetic field, and $\vec{k}$-direction is the photon's propagation direction.

When the free electron is in the high-intensity low-frequency laser field, the frequency of the radiation field is high enough to make the electron velocity relativistic, $\vec{F}_{B}$ cannot be ignored, the electron recoil is obvious 15-18. So when the free electron is in the low-intensity low-frequency field, although $\overrightarrow{F_{B}} \square \overrightarrow{F_{E}}, \overrightarrow{F_{B}}$ can be ignored, but $\overrightarrow{F_{B}}$ exists, Compton scattering exists, but it is too small to be observed and is ignored, the wavelength of scattered photon is almost the same, and the energy loss of the photon can be ignored. When photons interact with a large number of free electrons,

FEC effect appears: Photons to redshift and the photon beam to expand along the propagation direction.

Compton shift $\Delta \lambda=\lambda^{\prime}-\lambda=\frac{h}{m_{e} c}(1-\cos \theta)$, where $\lambda$ is the initial wavelength, $\lambda^{\prime}$ is the wavelength after scattering, $\Delta \lambda$ and $\theta$ are independent of the wavelength of the photon, $\Delta \lambda$ is only related to $\theta$. The initial velocity of the electron is ignored, the electron is completely free. Compton shift is inversely proportional to the mass of charged particles, Compton shift is mainly caused by the electrons. When a photon collides with a large number of electrons repeatedly, FEC redshift:

$$
Z_{F E C}=\sum z_{i}=\frac{N h}{\lambda m_{e} c}\langle 1-\cos \theta\rangle
$$

Where $z_{i}$ is the FEC redshift caused by the $\mathrm{i}$-th collision, $N$ is number of collisions; $\langle 1-\cos \theta\rangle$ is the average of $(1-\cos \theta)$. Eq. (1) is established under the condition that the wavelength change caused by the collision is negligible.

Since photon is plane electromagnetic wave ${ }^{15,16}$, the number of collisions $N$ is proportional to the wavelength $\lambda$ and optical path $D$ and electron density $n_{e}, N \propto \lambda D n_{e}$, let $K \propto \frac{h}{m_{e} c}\langle 1-\cos \theta\rangle$, we get:

$$
Z_{F E C}=K n_{e} D
$$

The condition for Eq. (2) to be satisfied is $Z_{F E C} \square 1$, moving to high redshifts $Z_{F E C}=\exp \left(K n_{e} D\right)-1$. 
That is to say, cosmological redshift and Hubble parameters are not only related to distance, but also related to corresponding electron density. Where $K$ (unit: $\mathrm{m}^{2}$ ) is a coefficient,

$$
K=f\left(n_{e}, T_{e}\right)
$$

When the electron density increases, the coupling force between electrons and other charged particles increases and $K$ decreases; when the electron temperature increases, the temperature of photon relative to electron decreases and $\mathrm{K}$ decreases. Eq. (3) is still unknown.

Since $\theta$ is independent of the wavelength of the photon, so $K$ and $Z_{F E C}$ are independent of the wavelength of the photon as well, similarly as for the Doppler effect, it is sometimes difficult to distinguish them.

\section{Evidence of FEC redshift in Flare redshift}

Flare redshift is a common phenomenon ${ }^{24-26}$ and it is difficult to explain with the Doppler effect, which is evidence of FEC redshift. The surface temperature of the Sun is $5700 \mathrm{~K}$ in the quiet Sun and almost all solar atmospheric spectral lines do not show any redshift ${ }^{27,28}$. The gravitational redshift of the Sun is known to be less than $1 \mathrm{~km} \mathrm{~S}^{-1}{ }^{29}$. When solar flares occur, their spectral profile has a redshift asymmetry that is difficult to explain with the Doppler effect. The redshift asymmetry rules out the Stark effect because Stark-induced shift occurs in dense plasma (plasma with electron concentration about and above $10^{16} \mathrm{~cm}^{-3} 30$ ). The electron density of solar flares is generally in the range of $10^{12} \square 10^{14} \mathrm{~cm}^{-3} 31,32$. When solar flares occur, the temperature of the solar atmosphere increases and ionization increases. A typical characteristic is that the spectral profile of the solar flare is significantly broadened and simultaneously redshifted. In flare AR 12205 ${ }^{24}$, there is a clear evidence of the relationship between the spectral broadening, the redshift, and FEC redshift. It excludes the Doppler effect.

In flare AR $12205^{24}$. The formation temperature of Si IV $1403 \AA, \mathrm{C}$ II $1335 \AA, \mathrm{Mg}$ II h $2803 \AA, \mathrm{H} \alpha 6563$, CA II K $3934 \AA$, CA II $8542 \AA$ in $\log T$ [k] is 4.8, 4.3, 4.0, 4.0, 4.0 and 4.0, from the highest to the lowest. The distance between the spectral line forming region and the core of the flare increases and the influence of the flare decreases. These are consistent with the spectral line width ${ }^{24}$.

The flare energy comes from the interior of the photosphere: (1) The flare erupts in the lower part of the chromosphere; (2) The fermi 29-31,50-102 keV erupts earlier than $17 \mathrm{GHz}$ (fast radio burst) for about $10 \mathrm{~s}$ at the highest point ${ }^{24}$, which indicates that the energy comes from fermi 29-31,50-102 keV. If the energy comes from the external high-energy electron beam, the electron beam will inevitably collide with the chromosphere particles during transportation to produce bremsstrahlung and heat the surrounding material, the $17 \mathrm{GHz}$ and 1-8 $\AA$ signals are earlier than for fermi $29-31,50-102 \mathrm{keV}$ and the trajectory of the electron beam should be observed before the flare eruption, but the electron trajectory has not been obtained ${ }^{24}$.

The radiation at 1-8 $\AA$ is caused by the free thermal electrons ${ }^{24}$. When the first burst of feimi $29-31 \mathrm{keV}$ $(\mathrm{T}=150-200 \mathrm{~s}$ ) occurred, there were no significant corresponding bursts at 1-8 $\AA$, due to the lack of free electrons in the chromosphere at the beginning of the flare. The radiation at 1-8 $\AA$ gradually increases when the thermal expansion, the number of free electrons, and the temperature of the electrons increase. When the second burst of fermi 29-31 keV ( $\mathrm{T}=240-360 \mathrm{~s}$ ) occurred, 1-8 $\AA$ followed fermi $29-31 \mathrm{keV}$, the spectrum lines Si IV $1403 \AA$, C II $1335 \AA$, Mg II h $2803 \AA$, H $66563 \AA$ began to broaden and redshift at the same time (See (Tei, A., et al. 2017) : Temporal evolution of the peak intensityError! Reference source not found.). The onset and the duration of the apparent broadening and redshift of these lines are consistent with the 1-8 $\AA$ radiation. This indicates that:(1) the apparent broadening and the redshift of these lines fully correlates with the increase number of free electrons and (2) the apparent broadening and redshift of these spectral lines is 
not caused by the Doppler effect from particle motion, but by FEC because the change in particle velocity caused by the thermal expansion takes time and the radiant ionization makes the free electrons increase immediately.

A possible explanation for the red asymmetry is proposed. After the flare occurs, the thermal expansion (the Balmer and $\mathrm{Ca}$ II $\mathrm{H}$ lines showed blue asymmetry Doppler effect ${ }^{24}$ ), and the number of free electrons gradually increases. The FEC effect also gradually increases, and the spectral linewidth gradually broadens ${ }^{24,25}$. When the FEC effect is larger than the Doppler effect, the blue asymmetry changes to a red asymmetry. The redshift occurs at the position where the intensity is highest and tracks the outer edge of the ribbon, which visually illustrates this phenomenon ${ }^{25}$. Similar phenomena include the high redshift of gamma-ray bursts ${ }^{33,34}$.

The FEC redshift and the Doppler effects were used to simulate the spectral line velocity in flare AR 12205. The electron density of the flare was in the range of $10^{12}-10^{14} \mathrm{~cm}^{-3}, \log T_{e}(\mathrm{k})=4-4.5^{31,32}$, the value of $K$ was roughly estimated around $2 \times 10^{-28} \mathrm{~m}^{2}$.

\section{Discussion}

The FEC redshift depends on the free electron fraction, is it constant in the Universe? On a large scale, CMB has a perfect blackbody radiation and an almost perfect isotropy ${ }^{35}$, indicating that the intergalactic medium has an almost uniform temperature and that the density of intergalactic electrons is almost uniform and isotropy as well. Dispersion measurements (DM) of fast radio bursts (FRB) also indicate that the density of intergalactic electrons is almost uniform on a large scale, and may be related to the redshift ${ }^{36-38}$. If the Hubble parameter not only depend on the distance fraction but free electron fraction then FEC redshift can perfectly explain why the Hubble parameter is isotropy but not perfect isotropy. Stars, red giants, Cepheid variable stars, supernovas, and quasars have a different atmospheric electron density, which leads to deviations in the Hubble parameter for different samples. A higher star mass results in a higher temperature and a larger the redshift. This is why the Hubble parameter measured based on CMB and the red giants and Cepheid variable stars are different. This is also why quasars are abnormally redshifted ${ }^{39,40}$ and why the Hubble parameter measured over a century is not "constant".

Because Eq. (3) is unknown, suppose the $K$ of the intergalactic electrons is the same as the $K$ of the flare electrons $K=2 \times 10^{-28} \mathrm{~m}^{2}$, the average electron density $\bar{n}_{e}$ obtained by this theory that produces $H_{0}(67.8$ $\mathrm{km} \mathrm{s}^{-1} \mathrm{Mpc}^{-1}$ ) is around $4 \times 10^{-5} \mathrm{~cm}^{-3}$, so the average electron density $\overline{n_{e}}$ is $\overline{n_{e}}<4 \times 10^{-5} \mathrm{~cm}^{-3}$. This value is much greater than the present value of the critical density, but if the Universe is infinite, then the critical density is meaningless. The most relevant intergalactic electron density obtained from the DM of fast radio bursts is about $10^{-7} \mathrm{~cm}^{-3}{ }^{36-38}$. The reason for the radiation broadening of DM is that the scattering is caused by a thin slab of fluctuating electron density ${ }^{36,41}$, therefore the value of $10^{-7} \mathrm{~cm}^{-3}$ is not the true intergalactic electron density, is greater than $10^{-7} \mathrm{~cm}^{-3}$. The interstellar electron density is within $1-10^{-3} \mathrm{~cm}^{-3} 36-38$. So $\overline{n_{e}}<4 \times 10^{-5} \mathrm{~cm}^{-3}$ is also possible.

\section{Conclusion}

The Compton effect of free electron and low-energy photons has been observed in the laboratory. As long as there is a reasonable density of free electrons in the Universe, the FEC effect will exist. FEC redshift and FEC cosmic model may work.

\section{Acknowledgments}


The author is grateful to Mark Krumholz for guidance, Edward L. Wright and A. Tei, et al. for sharing their knowledge and analyzing their observations.

\section{References:}

Rob Garner. Mystery of the universe's expansion rate widens with new Hubble data NASA's Goddard Space Flight Center, <https://www.nasa.gov/feature/goddard/2019/mystery-of-the-universe-s-expansion-rate-widens-with-new-hubbledata> (2019).

Castelvecchi, D. How fast is the Universe expanding? Cosmologists just got more confused, <https://www.nature.com/articles/d41586-019-02198-z> (2019).

Freedman, W. L. et al. The Carnegie-Chicago Hubble Program. VIII. An Independent Determination of the Hubble Constant Based on the Tip of the Red Giant Branch. The Astrophysical Journal 882, 34, doi:10.3847/1538-4357/ab2f73 (2019).

Marra, V., Amendola, L., Sawicki, I. \& Valkenburg, W. Cosmic variance and the measurement of the local Hubble parameter. Phys Rev Lett 110, 241305, doi:10.1103/PhysRevLett.110.241305 (2013).

Chen, G. C. F. et al. A SHARP view of HOLiCOW: HO from three time-delay gravitational lens systems with adaptive optics imaging. MNRAS 490, 1743, doi:10.1093/mnras/stz2547 (2019).

Riess, A. G., Casertano, S., Yuan, W., Macri, L. M. \& Scolnic, D. Large Magellanic Cloud Cepheid Standards Provide a 1\% Foundation for the Determination of the Hubble Constant and Stronger Evidence for Physics beyond $\wedge$ CDM. ApJ 876, 85, doi:10.3847/1538-4357/ab1422 (2019).

Migkas, K. et al. Probing cosmic isotropy with a new X-ray galaxy cluster sample through the LX-T scaling relation. Astronomy \& Astrophysics 636, doi:10.1051/0004-6361/201936602 (2020). Wright, E. L. Errors in Tired Light, <http://www.astro.ucla.edu/ wright/tiredlit.htm> (2008). Lubin, L. M. \& Sandage, A. The Tolman Surface Brightness Test for the Reality of the Expansion. IV. A Measurement of the Tolman Signal and the Luminosity Evolution of Early-Type Galaxies. The Astronomical Journal 122, 1084-1103, doi:10.1086/322134 (2001). Goldhaber, G. et al. Timescale Stretch Parameterization of Type la SupernovaB - Band Light Curves. The Astrophysical Journal 558, 359-368, doi:10.1086/322460 (2001). Blondin, S. et al. Time Dilation in Type la Supernova Spectra at High Redshift. The Astrophysical Journal 682, 724-736, doi:10.1086/589568 (2008).

Sandage, A. \& Lubin, L. M. The Tolman Surface Brightness Test for the Reality of the Expansion. I. Calibration of the Necessary Local Parameters. The Astronomical Journal 121, 2271-2288, doi:10.1086/320394 (2001). Carlstrom, J. E., Holder, G. P. \& Reese, E. D. Cosmology with the Sunyaev-Zel'dovich Effect. Annual Review of Astronomy and Astrophysics 40, 643-680, doi:10.1146/annurev.astro.40.060401.093803 (2002).

Malu, S. et al. Relativistic inverse Compton scattering of photons from the early universe. Sci Rep 7, 16918, doi:10.1038/s41598-017-17104-8 (2017).

Brown, L. S. \& Kibble, T. W. B. Interaction of Intense Laser Beams with Electrons. Phys. Rev. 133, A705, doi:10.1103/Phys. Rev. 133, A705 (1964). 
Moore, C. I. Observation of the Transition from Thomson to Compton Scattering in Optical Multiphoton Interactions with Electrons. Phys Rev Lett 74, 2439, doi:10.1103/PhysRevLett.77.2334 (1995).

Babzien, M. et al. Observation of the second harmonic in Thomson scattering from relativistic electrons. Phys Rev Lett 96, 054802, doi:10.1103/PhysRevLett.96.054802 (2006).

Lotstedt, E. \& Jentschura, U. D. Nonperturbative Treatment of Double Compton Backscattering in Intense Laser Fields. Phys Rev Lett 103, 110404, doi:10.1103/PHYSREVLETT.103.110404 (2009).

Zhang, B. et al. Quantum Mechanisms of Electron and Positron Acceleration through Nonlinear Compton Scatterings and Nonlinear Breit-Wheeler Processes in Coherent Photon Dominated Regime. Sci Rep 9, 18876, doi:10.1038/s41598019-55472-5 (2019).

Fixsen, D. J. et al. The Cosmic Microwave Background Spectrum from the FullCOBEFIRAS Data Set. The Astrophysical Journal 473, 576-587, doi:10.1086/178173 (1996).

Noterdaeme, P., Ledoux, C., Srianand, R., Petitjean, P. \& Lopez, S. Diffuse molecular gas at high redshift. Astronomy \& Astrophysics 503, 765-770, doi:10.1051/0004-6361/200912330 (2009).

Noterdaeme, P., Petitjean, P., Srianand, R., Ledoux, C. \& López, S. The evolution of the cosmic microwave background temperature. Astronomy \& Astrophysics 526, doi:10.1051/0004-6361/201016140 (2011).

Wright, E. L. Can the CMBR be redshifted starlight? NO!, <http://www.astro.ucla.edu/ wright/stars vs cmb.html> (1997).

Tei, A. et al. Blue-wing enhancement of the chromospheric $\mathrm{Mg}$ ii $\mathrm{h}$ and $\mathrm{k}$ lines in a solar flare. Publications of the Astronomical Society of Japan 70, doi:10.1093/pasj/psy047 (2018).

Kerr, G. S., Simões, P. J. A., Qiu, J. \& Fletcher, L. IRIS observations of the Mg ii h and k lines during a solar flare. A\&A $\mathbf{5 8 2}$ A50, doi:10.1051/0004-6361/201526128 (2015).

Jing, J. et al. Unprecedented Fine Structure of a Solar Flare Revealed by the $1.6 \mathrm{~m}$ New Solar Telescope. Sci Rep 6, 24319, doi:10.1038/srep24319 (2016).

Lemaire, P., Vial, J. C., Curdt, W., Schühle, U. \& Wilhelm, K. Hydrogen Ly- $\alpha$ and Ly- $\beta$ full Sun line profiles observed with SUMER/SOHO (1996-2009). A\&A 581, A26, doi:10.1051/0004-6361/201526059 (2015).

Brown, S. A., Fletcher, L. \& Labrosse, N. Doppler speeds of the hydrogen Lyman lines in solar flares from EVE. A\&A 596, A51, doi:10.1051/0004-6361/201628390 (2016).

Joyce, S. R. G. et al. The gravitational redshift of Sirius B. MNRAS 481, 2361, doi:10.1093/mnras/sty2404 (2018). Halenka, J., Olchawa, W., Madej, J. \& Grabowski, B. Pressure Shift and Gravitational Redshift of Balmer Lines in White Dwarfs: Rediscussion. ApJ 808, 131, doi:10.1088/0004-637x/808/2/131 (2015).

Kerr, G. S., Carlsson, M., Allred, J. C., Young, P. R. \& Daw, A. N. SI iv Resonance Line Emission during Solar Flares: Non-LTE, Nonequilibrium, Radiation Transfer Simulations. ApJ 871, 23, doi:10.3847/1538-4357/aaf46e (2019).

Reep, J. W., Bradshaw, S. J., Crump, N. A. \& Warren, H. P. Efficient Calculation of Non-local Thermodynamic Equilibrium Effects in Multithreaded Hydrodynamic Simulations of Solar Flares. ApJ 871, 18, doi:10.3847/1538-4357/aaf580 (2019). Choudhury, T. R. \& Srianand, R. Probing the dark ages with redshift distribution of gamma-ray bursts. MNRAS 336, L27, doi:10.1046/j.1365-8711.2002.05984.x (2002). 

Gamma-ray Bursts: prospects for observations with future X-ray instruments. MNRAS 410, 1611, doi:10.1111/j.13652966.2010.17540.x (2011).

35

36

37

Ben-Dayan, I., Durrer, R., Marozzi, G. \& Schwarz, D. J. Value of H(0) in the inhomogeneous universe. Phys Rev Lett 112, 221301, doi:10.1103/PhysRevLett.112.221301 (2014).

Katz, J. I. Inferences from the Distributions of Fast Radio Burst Pulse Widths, Dispersion Measures, and Fluences. ApJ 818, 19, doi:10.3847/0004-637x/818/1/19 (2016).

Yao, J. M., Manchester, R. N. \& Wang, N. A New Electron-Density Model for Estimation of Pulsar and Frb Distances. ApJ 835, 29, doi:10.3847/1538-4357/835/1/29 (2017).

Ashmore, L. A Relationship between Dispersion Measure and Redshift Derived in Terms of New Tired Light. JHEPGC 02, 512, doi:10.4236/jhepgc.2016.24045 (2016).

Dai, D. C., Starkman, G. D., Stojkovic, B., Stojkovic, D. \& Weltman, A. Using quasars as standard clocks for measuring cosmological redshift. Phys Rev Lett 108, 231302, doi:10.1103/PhysRevLett.108.231302 (2012).

Chu, Y. Q. On the relationship between redshift and distance. Nature magazine(China) 5-4, 364, doi:CNKI:SUN:ZRZZ.0.1981-05-013 (1981).

Williamson, I. P. Pulse Broadening due to Multiple Scattering in the Interstellar Medium. MNRAS 157, 55, doi:10.1093/mnras/157.1.55 (1972). 